\title{
Combination of sacral-alar-iliac screw and cortical bone trajectory screw techniques for lumbosacral fixation: technical note
}

\author{
Keitaro Matsukawa, MD, PhD, ${ }^{1}$ Takashi Kato, MD, ${ }^{1}$ Ralph Mobbs, MD, FRACS, ${ }^{2}$ \\ Yoshiyuki Yato, MD, PhD, 1 and Takashi Asazuma, MD, PhD' \\ 1Department of Orthopaedic Surgery, National Hospital Organization, Murayama Medical Center, Musashimurayama, Tokyo, \\ Japan; and 2Department of Neurosurgery, Prince of Wales Private Hospital, Randwick, Sydney, Australia
}

\begin{abstract}
OBJECTIVE Lumbosacral fixation plays an important role in the management of devastating spinal pathologies, including osteoporosis, fracture, infection, tumor resection, and spinal deformities, which require long-segment fusion constructs to the sacrum. The sacral-alar-iliac (SAI) screw technique has been developed as a promising solution to facilitate both minimal invasiveness and strong fixation. The rationale for SAI screw insertion is a medialized entry point away from the ilium and in line with cranial screws. The divergent screw path of the cortical bone trajectory (CBT) provides a higher amount of cortical bone purchase and strong screw fixation and has the potential to harmoniously align with SAI screws due to its medial starting point. However, there has been no report on the combination of these two techniques. The objective of this study was to assess the feasibility of this combination technique.
\end{abstract}

METHODS The subjects consisted of 17 consecutive patients with a mean age of $74.2 \pm 4.7$ years who underwent posterior lumbosacral fixation for degenerative spinal pathologies using the combination of SAI and CBT fixation techniques. There were 8 patients with degenerative scoliosis, 7 with degenerative kyphosis, 1 with an osteoporotic vertebral fracture at L5, and 1 with vertebral metastasis at L5. Fusion zones included T10-sacrum in 13 patients, L2-sacrum in 2, and L4-sacrum in 2.

RESULTS No patients required complicated rod bending or the use of a connector for rod assembly in the lumbosacral region. Postoperative CT performed within a week after surgery showed that all lumbosacral screws were in correct positions and there was no incidence of neurovascular injuries. The lumbosacral bone fusion was confirmed in $81.8 \%$ of patients at 1-year follow-up based on fine-cut CT scanning. No patient showed a significant loss of spinal alignment or rod fracture in the lumbosacral transitional region.

CONCLUSIONS This is the first paper on the feasibility of a combination technique using SAI and CBT screws. This technique could be a valid option for lumbosacral fixation due to the ease of rod placement with potential reductions in operative time and blood loss.

https://thejns.org/doi/abs/10.3171/2020.1.SPINE191420

KEYWORDS sacral-alar-iliac screw; cortical bone trajectory; lumbosacral fixation; long fusion; screw fixation; minimal invasiveness; lumbar; sacral; surgical technique

$\mathrm{T}$ HeRE is a growing demand for rigid lumbosacral fixation due to an increase in varieties of spinal pathologies in the aging population. From an anatomical point of view, the lumbosacral junction is one of the challenging spinal regions because of significant mechanical forces, poor sacral bone quality, complex anatomy, and proximity to major visceral structures, often leading to pseudarthrosis, hardware failure, and loss of correction. ${ }^{1}$
Especially in the surgical management of adult spinal deformity, achieving sagittal global alignment through realizing spinopelvic harmony is essential for improving the quality of life, and surgeons need to achieve sacral anchoring for satisfactory resistance to cantilever force. ${ }^{2}$

To improve anchoring to the lumbosacral region, the sacral-alar-iliac (SAI) screw technique has been introduced in both pediatric and adult populations. ${ }^{3,4}$ The path

ABBREVIATIONS CBT = cortical bone trajectory; $\mathrm{C7SVA}=\mathrm{C7}$ sagittal vertical axis; $\mathrm{LL}=$ lumbar lordosis; $\mathrm{PI}=$ pelvic incidence; $\mathrm{PT}=$ pelvic tilt; $\mathrm{SAI}=$ sacral-alar-iliac; $\mathrm{SI}=$ sacroiliac.

SUBMITTED November 25, 2019. ACCEPTED January 20, 2020.

INCLUDE WHEN CITING Published online March 20, 2020; DOI: 10.3171/2020.1.SPINE191420. 
of SAI screws starts from a medialized point away from the iliac crest, crosses the sacroiliac (SI) joint (therefore gripping the double cortical layer), and extends sufficiently anterolateral to the sacral pivot point, advocated by McCord et al. ${ }^{5}$ allowing for the placement of longer and larger screws. ${ }^{6}$ Compared with classic iliac screws, SAI screws are less prominent, requiring significantly reduced muscle dissection and showing superior biomechanical performance. ${ }^{78}$ Some authors have reported the clinical outcomes using SAI screws, including lower rates of reoperation, surgical site infection, and symptomatic screw prominence. ${ }^{9,10}$

Similar to this novel SI fixation technique, during the last decade, the cortical bone trajectory (CBT) showed increasing popularity as a new alternative to the conventional transpedicular trajectory. ${ }^{11,12}$ Biomechanical studies have shown that CBT screws can achieve superior fixation in comparison with conventional pedicle screws by engagement with denser regions within the vertebra. ${ }^{12,13}$ Another benefit of using the CBT is its minimal invasiveness due to a reduced need for muscle dissection and preservation of the superior adjacent facet joints because CBT screws can have a divergent trajectory from a more medial and caudal starting point. ${ }^{14-16}$ Recent clinical studies have demonstrated that the CBT technique is significantly less invasive and leads to improved clinical outcomes compared with the conventional technique. ${ }^{17-19}$

These innovative spinal instrumentation techniques have been developed as promising solutions for both screw fixation and minimal invasiveness; however, to the best of our knowledge, there has been no report on the combination of these two SAI and CBT fixation techniques in clinical practice. One of the benefits of this combination is that their entry points are relatively medial in position, and harmoniously in line so as to avoid technical problems for rod placement, such as the need for complicated rod bending and use of an offset connector. The objective of this study was to report a case series to assess the feasibility of the combination of SAI and CBT fixation techniques.

\section{Methods}

A total of 17 consecutive patients ( 3 men and 14 women; mean age $74.2 \pm 4.7$ years, range $65-81$ years) underwent posterior lumbosacral fixation for degenerative spinal pathologies using the combination of SAI and CBT fixation techniques from June 2017 to June 2019. The mean duration of follow-up was 13.8 months (range 4-24 months). There were 8 patients with degenerative scoliosis, 7 with degenerative kyphosis, 1 with an osteoporotic vertebral fracture at L5, and 1 with vertebral metastasis at L5. Fusion zones included T10-sacrum in 13 patients, L2-sacrum in 2, and L4-sacrum in 2.

\section{Surgical Procedure}

Through a midline incision over the corresponding vertebrae, the paraspinal muscles were dissected to expose the entry points of the SAI and CBT screws. During the procedure, the surgeons verified the accuracy of the entry point position and trajectory direction for screw placement using fluoroscopic support (Fig. 1). The starting point of

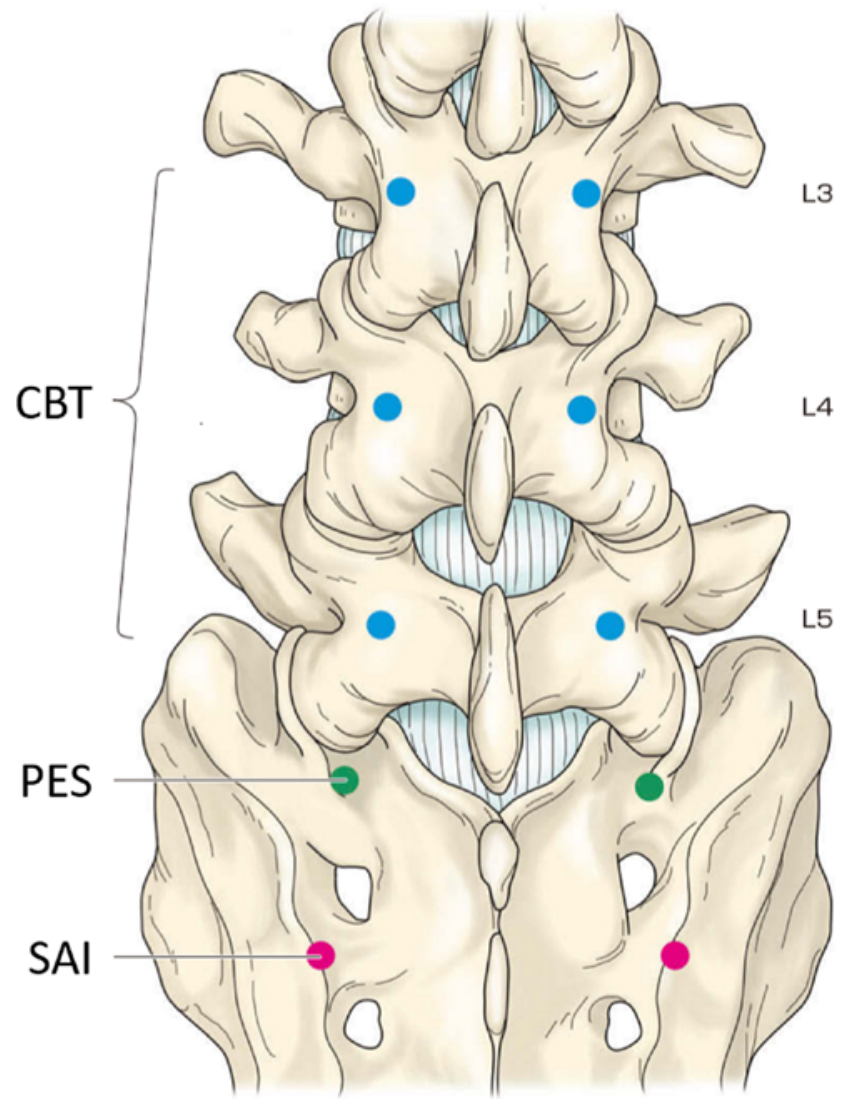

FIG. 1. Schematic of entry points for CBT, penetrating endplate, and SAI screws demonstrating harmoniously in-line positions. PES = penetrating endplate screw. Copyright Medical View Co., Ltd. Published with permission. Figure is available in color online only.

the SAI screw is $1 \mathrm{~mm}$ lateral and $1 \mathrm{~mm}$ inferior to the $\mathrm{S} 1$ dorsal foramen, ${ }^{8}$ which is harmoniously in line with the entry points of cranial screws. The SAI screw trajectory was directed so as to penetrate the cortical wall of the SI joint through the superior rim of the sciatic notch toward the anterior inferior iliac spine. In accordance with a previous morphometric study, the entry point for lumbar CBT was made at the lateral aspect of the pars interarticularis, which corresponded to a 5- or 7-o'clock orientation in the pedicle. ${ }^{20}$ The trajectory was directed about $20^{\circ}-25^{\circ}$ cranially and $10^{\circ}$ laterally along the inferior border of the pedicle toward the posterior half of the superior endplate. For the thoracic spine, less steep and less divergent screw trajectories were made..$^{21,22}$ Similarly, the sacral CBT technique was adopted to engage with denser sacral bone. The trajectory started at more medial points than that of the conventional sacral pedicle screw technique, was directed straight forward in the axial plane without convergence, and penetrated the middle of the sacral endplate. ${ }^{23}$ The bilateral rods were bent into an ideal sagittal contour to correct the deformity and were initially connected with S1 and SAI screws. Then, the rods were applied to thoracolumbar screws from caudal to cranial using a dual-cantilever maneuver. The supplemental use of sublaminar taping was applied proximal to the upper instrumented vertebra 

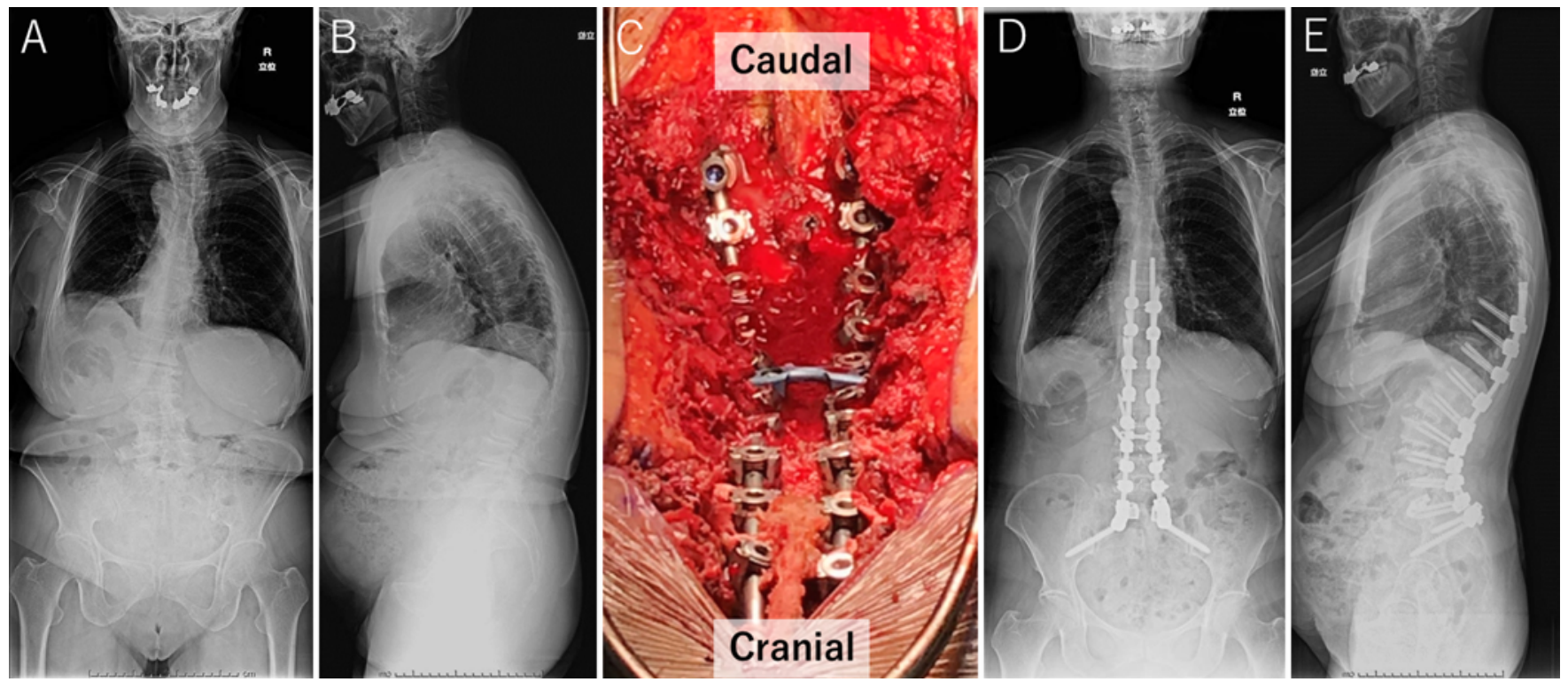

FIG. 2. Case 1. An 80-year-old woman with degenerative kyphosis underwent spinal fusion from T10 to the sacrum. A and B: Preoperative anteroposterior $(A)$ and lateral $(B)$ standing whole-spine radiographs showing lumbar kyphosis and pelvic retroversion

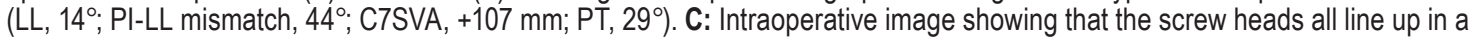
straight line. The proximal rod length is long enough to place sublaminar taping from T9 (1 level proximal to the upper instrumented vertebra) to T11. D and E: Postoperative anteroposterior (D) and lateral (E) whole-spine radiographs obtained 1.5 years postoperatively, showing improved global sagittal alignment (LL, $56^{\circ} ; \mathrm{PI}-\mathrm{LL}$ mismatch, $-8^{\circ} ; \mathrm{C} 7 \mathrm{SVA},+23 \mathrm{~mm} ; \mathrm{PT}, 13^{\circ}$ ). Figure is available in color online only.

to prevent screw pullout and mitigate adjacent-segment stress if necessary. ${ }^{24}$ All patients received posterior lumbar interbody fusion for the L5-S1 segment. Lumbosacral bone fusion assessment was made using CT performed 1 year postoperatively with coronal and sagittal reconstruction according to an evaluation described by Ito et al. ${ }^{25}$

\section{Results}

In this case series, no patients required complicated rod bending or the use of a connector for rod assembly in the lumbosacral region. Postoperative CT performed within a week after surgery showed that all lumbosacral screws were in correct positions and there was no incidence of neurovascular injuries. No patients were lost to followup. Excluding 6 patients with follow-up < postoperative 6 months, 11 of the 17 patients were followed for $>1$ year. Among those, lumbosacral bone fusion was confirmed in 9 patients $(81.8 \%)$, and 2 patients $(18.2 \%)$ showed partial fusion with no evidence of pseudarthrosis. No patient showed significant loss of spinal alignment or rod fracture in the lumbosacral transitional region.

\section{Illustrative Cases}

Case 1

An 80-year-old woman presented with complaints of low-back pain and difficulty standing for less than 5 minutes. Preoperative standing whole-spine radiographs showed lumbar kyphosis (lumbar lordosis [LL], 14º pelvic incidence $[\mathrm{PI}]-\mathrm{LL}$ mismatch, $44^{\circ} ; \mathrm{C} 7$ sagittal vertical axis [C7SVA], $+107 \mathrm{~mm}$; pelvic tilt [PT], 29 ${ }^{\circ}$ ) (Fig. 2A and B). Posterior spinal fusion was performed from T10 to the sa- crum combined with sublaminar taping for T9-11, lateral lumbar interbody fusion from L2-3 to L4-5, and posterior lumbar interbody fusion for L5-S1 (Fig. 2C). The standing radiographs obtained 1.5 years postoperatively showed improved global sagittal alignment (LL, 56 $6^{\circ}$ PI-LL mismatch, $-8^{\circ}$; C7SVA, $+23 \mathrm{~mm}$; PT, $13^{\circ}$ ) (Fig. 2D and E).

\section{Case 2}

A 68-year-old man presented with low-back pain and bilateral sciatica. Consecutive treatment was unsuccessful. Preoperative standing whole-spine radiographs showed degenerative lumbar kyphoscoliosis (coronal Cobb angle of L1-4, $67^{\circ}$; LL, $16^{\circ}$; PI-LL mismatch, $37^{\circ}$; C7SVA, +62 $\mathrm{mm} ; \mathrm{PT}, 25^{\circ}$ ) (Fig. 3A and B). Anteroposterior spinal fusion was performed from T10 to the sacrum following the same procedures as in the previous case. Global spinal alignment was corrected (Cobb angle of L1-4, 12 ${ }^{\circ}$; LL, 58 ${ }^{\circ}$ PI-LL mismatch, $-5^{\circ}$; C7SVA, $\left.-10 \mathrm{~mm} ; \mathrm{PT}, 19^{\circ}\right)$. He did not complain of any low-back or leg pain at the 1-year follow-up (Fig. 3C and D).

\section{Discussion}

Lumbosacral fixation plays an important role in the management of devastating spinal pathologies, including osteoporosis, fracture, infection, tumor resection, and spinal deformities that may require long-segment fusion constructs to the sacrum. This is the first paper to report on the feasibility of a novel technique using the combination of SAI and CBT screw fixation.

To obtain appropriate biomechanical anchoring, SAI screws should traverse sacral bone, have cortical pur- 

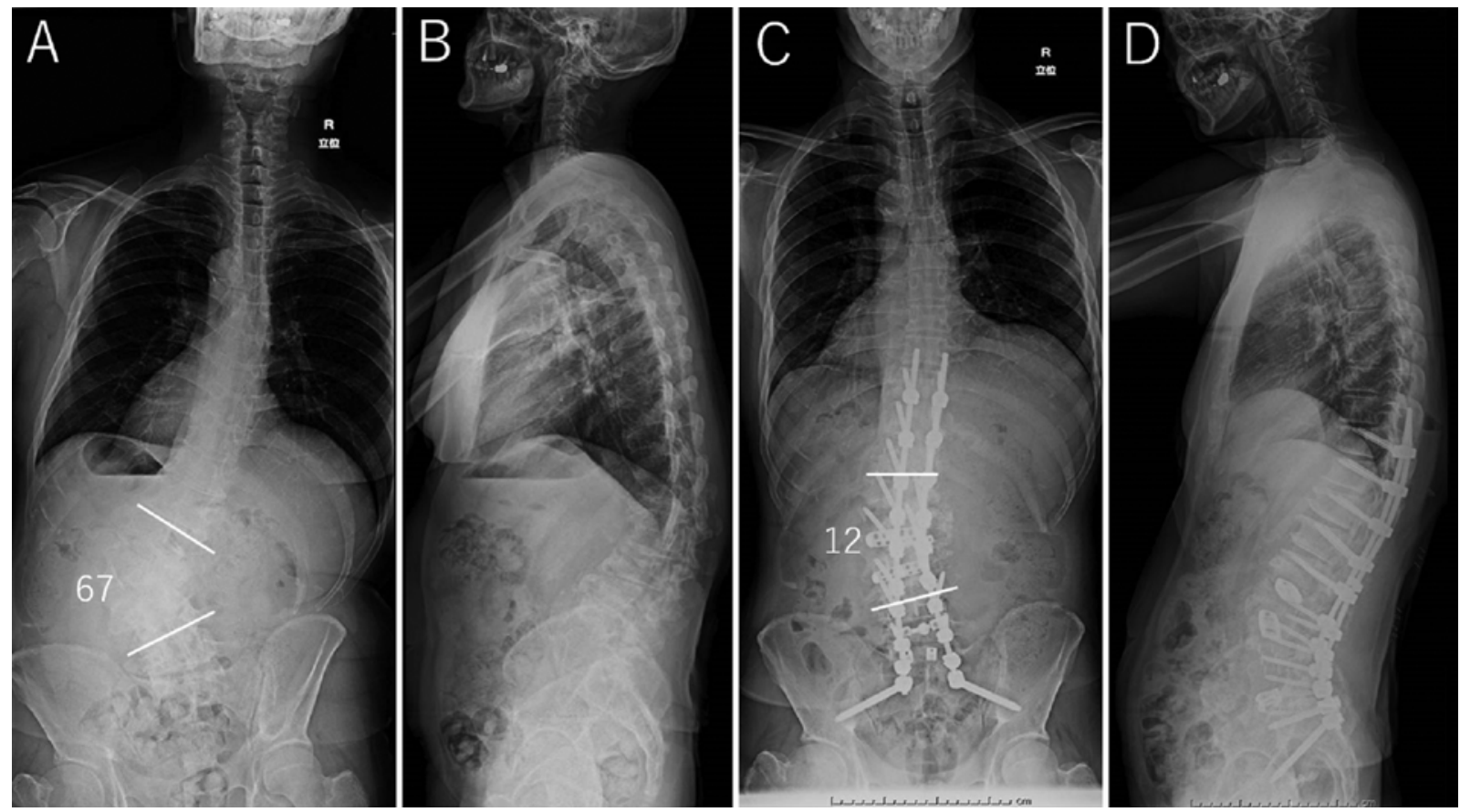

FIG. 3. Case 2. A 68-year-old man with degenerative kyphoscoliosis underwent spinal fusion from T10 to the sacrum. A and B: Preoperative anteroposterior $(A)$ and lateral $(B)$ standing whole-spine radiographs showing lumbar scoliosis with kyphosis

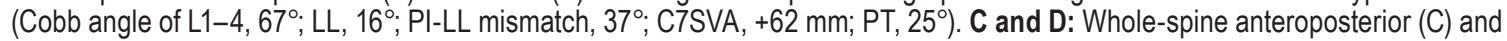
lateral (D) radiographs obtained 1 year postoperatively, showing improved global coronal and sagittal alignment (Cobb angle of

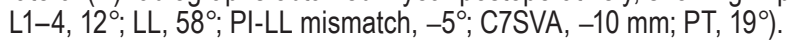

chase in the SI joint, go as far anteriorly and laterally from the sacral pivot point as possible, ${ }^{5}$ and end between the inner iliac cortices, which necessitates a medial entry point. In terms of the entry point of the SAI trajectory, several different points have been reported, such as $1 \mathrm{~mm}$ lateral and $1 \mathrm{~mm}$ inferior to the $\mathrm{S} 1$ dorsal foramen, ${ }^{8}$ the junction of the lateral sacral crest and the midpoint between the $\mathrm{S} 1$ and S2 dorsal foramina, ${ }^{26}$ and $2 \mathrm{~mm}$ medial to the apex of the lateral sacral crest on the midline between the S1

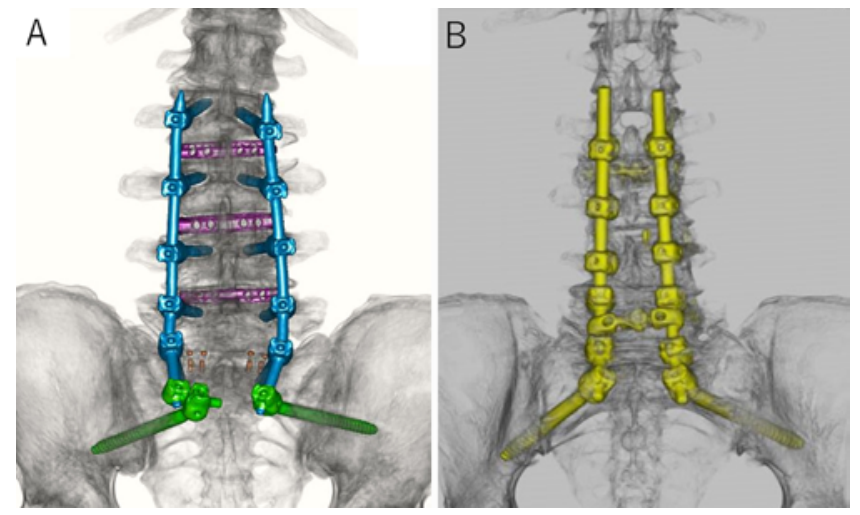

FIG. 4. Comparison of the combination with SAI screws between the conventional pedicle screw technique and CBT technique. A: Combination with the conventional technique, necessitating medial rod bending of the distal end (right side) and use of an off-set connector (left side). $\mathrm{B}$ : Combination with the CBT technique, showing straight rods for ease of implantation. Figure is available in color online only. and $\mathrm{S} 2$ dorsal foramina. ${ }^{27}$ Contrary to the iliac screw technique, there is extensive literature stating that the SAI medialized entry points are harmoniously located with cranial entry points of conventional pedicle screws. However, surgeons frequently have difficulty with rod application in this region because the SAI screw relies on too medial an entry point to connect with conventional cranial pedicle screws. This tendency is prominent in patients with a medialized iliac crest, short anteroposterior diameter of the SI joint, and coronalization of the SI joint. Examples of clinical ingenuity for easy rod assembly include medial curvature of the distal end of the rod, S1 pedicle screw insertion from a medial entry point, and the use of an offset connector (Fig. 4A).

Compared with the conventional technique, the most practical advantage of using the combination of SAI and CBT screws is technical ease of rod insertion because these entry points are anatomically in line (Fig. 4B). ${ }^{8,20,23}$ It can eliminate the need for biplanar rod contouring and connector usage with potential reductions of the operative time and blood loss. It can also minimize the risk of rod fracture because intraoperatively bending the rod multiple times to fit the screws can weaken the mechanical properties of the rod. ${ }^{28}$ Furthermore, this combination technique provides two major advantages in addition to simple screw-rod assembly. First, the medial position of entry points and divergent trajectory of both SAI and CBT screws lead to less lateral muscle dissection and reduce the approach-related morbidity. This could minimize ischemic necrosis and denervation of the posterior muscles, reduce postoperative low-back pain, and facilitate postop- 
erative rehabilitation and early mobilization. Second, both SAI and CBT screws are characterized by contacting with a high level of purchase in the cortical bone. Contrary to iliac screws anchored in all cancellous beds, SAI screws show cortical purchase in the SI joint and show superior biomechanical performance in the spinal construct. ${ }^{8}$ Similarly, CBT utilizes a craniolaterally directed path through the pedicle so that screw threads contact with the highest density of cortical bone, especially from the entry point of the pars interarticularis to the inferior border of the pedicle. A sufficient number of basic studies on CBT have shown the greater bone density of the trajectory and stronger mechanical resistance to pullout and against toggle loading than the traditional pedicle screw trajectory. ${ }^{12,13} \mathrm{~A}$ combination of SAI and CBT fixation techniques could provide a rigid construct across the lumbosacral junction and could be a valid treatment option for adult patients with spinal deformity.

One of the concerns regarding this combined technique is the need for fluoroscopic or navigation assistance to appropriately place the screws because of the narrow bony corridors to achieve adequate screw fixation. From the same point of view, it also necessitates high-level surgical skill. However, some modern technology, including 3D image guidance systems, patient-specific guide systems, and robotic guidance, has recently been introduced to improve screw placement accuracy with less intraoperative radiation exposure. ${ }^{29,30}$ Based on the clinical experience of this case series, this combination technique may be an appropriate alternative to the conventional lumbosacral fixation technique. Further biomechanical and clinical studies are needed to confirm the usefulness of this combination technique.

\section{Conclusions}

To the best of our knowledge, this is the first paper on the feasibility of a combination technique using SAI and CBT screws. This technique could be a valid option for lumbosacral fixation due to the ease of rod placement and has the potential to become an effective solution in terms of screw fixation and minimal invasiveness.

\section{References}

1. Kim YJ, Bridwell KH, Lenke LG, et al. Pseudarthrosis in long adult spinal deformity instrumentation and fusion to the sacrum: prevalence and risk factor analysis of 144 cases. Spine (Phila Pa 1976). 2006;31(20):2329-2336.

2. Schwab FJ, Blondel B, Bess S, et al. Radiographical spinopelvic parameters and disability in the setting of adult spinal deformity: a prospective multicenter analysis. Spine (Phila Pa 1976). 2013;38(13):E803-E812.

3. Chang TL, Sponseller PD, Kebaish KM, Fishman EK. Low profile pelvic fixation: anatomic parameters for sacral alariliac fixation versus traditional iliac fixation. Spine (Phila Pa 1976). 2009;34(5):436-440.

4. Sponseller PD, Zimmerman RM, Ko PS, et al. Low profile pelvic fixation with the sacral alar iliac technique in the pediatric population improves results at two-year minimum follow-up. Spine (Phila Pa 1976). 2010;35(20):1887-1892.

5. McCord DH, Cunningham BW, Shono Y, et al. Biomechanical analysis of lumbosacral fixation. Spine (Phila Pa 1976). 1992;17(8)(suppl):S235-S243.
6. Tavares Junior MCM, de Souza JPV, Araujo TPF, et al. Comparative tomographic study of the S2-alar-iliac screw versus the iliac screw. Eur Spine J. 2019;28(4):855-862.

7. Cunningham BW, Sponseller PD, Murgatroyd AA, et al. A comprehensive biomechanical analysis of sacral alar iliac fixation: an in vitro human cadaveric model. J Neurosurg Spine. 2019;30(3):367-375.

8. O'Brien JR, Yu W, Kaufman BE, et al. Biomechanical evaluation of S2 alar-iliac screws: effect of length and quad-cortical purchase as compared with iliac fixation. Spine (Phila Pa 1976). 2013;38(20):E1250-E1255.

9. Elder BD, Ishida W, Lo SL, et al. Use of S2-alar-iliac screws associated with less complications than iliac screws in adult lumbosacropelvic fixation. Spine (Phila $\mathrm{Pa}$ 1976). 2017;42(3):E142-E149.

10. Ilyas H, Place H, Puryear A. A comparison of early clinical and radiographic complications of iliac screw fixation versus $\mathrm{S} 2$ alar iliac (S2AI) fixation in the adult and pediatric populations. J Spinal Disord Tech. 2015;28(4):E199-E205.

11. Mobbs RJ. The "medio-latero-superior trajectory technique": an alternative cortical trajectory for pedicle fixation. Orthop Surg. 2013;5(1):56-59.

12. Santoni BG, Hynes RA, McGilvray KC, et al. Cortical bone trajectory for lumbar pedicle screws. Spine J. 2009;9(5):366373.

13. Baluch DA, Patel AA, Lullo B, et al. Effect of physiological loads on cortical and traditional pedicle screw fixation. Spine (Phila Pa 1976). 2014;39(22):E1297-E1302.

14. Hung CW, Wu MF, Hong RT, et al. Comparison of multifidus muscle atrophy after posterior lumbar interbody fusion with conventional and cortical bone trajectory. Clin Neurol Neurosurg. 2016;145:41-45.

15. Khanna N, Deol G, Poulter G, Ahuja A. Medialized, musclesplitting approach for posterior lumbar interbody fusion: technique and multicenter perioperative results. Spine (Phila Pa 1976). 2016;41(suppl 8):S90-S96.

16. Matsukawa K, Kato T, Yato Y, et al. Incidence and risk factors of adjacent cranial facet violation following pedicle screw insertion using cortical bone trajectory technique. Spine (Phila Pa 1976). 2016;41(14):E851-E856.

17. Lee GW, Son JH, Ahn MW, et al. The comparison of pedicle screw and cortical screw in posterior lumbar interbody fusion: a prospective randomized noninferiority trial. Spine J. 2015;15(7):1519-1526.

18. Sakaura H, Miwa T, Yamashita T, et al. Posterior lumbar interbody fusion with cortical bone trajectory screw fixation versus posterior lumbar interbody fusion using traditional pedicle screw fixation for degenerative lumbar spondylolisthesis: a comparative study. J Neurosurg Spine. 2016;25(5):591-595.

19. Takenaka S, Mukai Y, Tateishi K, et al. Clinical outcomes after posterior lumbar interbody fusion: comparison of cortical bone trajectory and conventional pedicle screw insertion. Clin Spine Surg. 2017;30(10):E1411-E1418.

20. Matsukawa K, Yato Y, Nemoto O, et al. Morphometric measurement of cortical bone trajectory for lumbar pedicle screw insertion using computed tomography. J Spinal Disord Tech. 2013;26(6):E248-E253.

21. Matsukawa K, Yato Y, Hynes RA, et al. Cortical bone trajectory for thoracic pedicle screws: a technical note. Clin Spine Surg. 2017;30(5):E497-E504.

22. Xuan J, Zhang D, Jin HM, et al. Minimally invasive cortical bone trajectory screws placement via pedicle or pedicle rib unit in the lower thoracic spine: a cadaveric and radiographic study. Eur Spine J. 2016;25(12):4199-4207.

23. Matsukawa K, Yato Y, Kato T, et al. Cortical bone trajectory for lumbosacral fixation: penetrating S-1 endplate screw technique: technical note. J Neurosurg Spine. 2014;21(2):203-209. 
24. Viswanathan VK, Ganguly R, Minnema AJ, et al. Biomechanical assessment of proximal junctional semi-rigid fixation in long-segment thoracolumbar constructs. J Neurosurg Spine. 2018;30(2):184-192.

25. Ito Z, Matsuyama Y, Sakai Y, et al. Bone union rate with autologous iliac bone versus local bone graft in posterior lumbar interbody fusion. Spine (Phila Pa 1976). 2010;35(21):E1101-E1105.

26. Mattei TA, Fassett DR. Combined S-1 and S-2 sacral alariliac screws as a salvage technique for pelvic fixation after pseudarthrosis and lumbosacropelvic instability: technical note. J Neurosurg Spine. 2013;19(3):321-330.

27. Yamada K, Higashi T, Kaneko K, et al. Optimal trajectory and insertion accuracy of sacral alar iliac screws. Acta Orthop Traumatol Turc. 2017;51(4):313-318.

28. Lindsey C, Deviren V, Xu Z, et al. The effects of rod contouring on spinal construct fatigue strength. Spine. 2006;31(15):1680-1687.

29. Bederman SS, Hahn P, Colin V, et al. Robotic guidance for $\mathrm{S} 2$-alar-iliac screws in spinal deformity correction. Clin Spine Surg. 2017;30(1):E49-E53.

30. Matsukawa K, Kaito T, Abe Y. Accuracy of cortical bone trajectory screw placement using patient-specific template guide system [published online July 3, 2019]. Neurosurg Rev. doi:10.1007/s10143-019-01140-1

\section{Disclosures}

Dr. Mobbs: direct stock ownership in Medtronic, and royalties from Stryker and A-Spine ASIA.

\section{Author Contributions}

Conception and design: Matsukawa. Acquisition of data: Matsukawa, Kato. Analysis and interpretation of data:

Matsukawa. Drafting the article: Matsukawa. Critically revising the article: Mobbs, Yato, Asazuma. Reviewed submitted version of manuscript: Mobbs, Yato, Asazuma. Approved the final version of the manuscript on behalf of all authors: Matsukawa. Administrative/technical/material support: Matsukawa. Study supervision: Mobbs, Yato, Asazuma.

\section{Correspondence}

Keitaro Matsukawa: National Hospital Organization, Murayama Medical Center, Tokyo, Japan. keitaro197897@hotmail.com. 